\title{
Interleukin-34 is associated with hepatocellular carcinoma in chronic hepatitis $B$ patients
}

\section{Kehui Liu}

Department of infectious Disease, Ruijin Hospital, Shanghai Jiaotong University School of Medicine

\section{Yezhou Ding}

Department of infectious Diseases, Ruijin Hospital, Shanghai Jiaotong University School of Medicine

\section{Yun Wang}

Departmen of Infectious Diseases, Ruijin Hospital, Shanghai Jiaotong University School of Medicine

\section{Qingqing Zhao}

Department of Infectious Disease, Ruijin Hospital, Shanghai Jiaotong University School of Medicine

\section{Lei Yan}

Department of Infectious Disease, Ruijin Hospital, Shanghai Jiaotong University School of Medicine Jingdong Xie

Department of Infectious Diseases, Ruijin Hospital, Shanghai Jiaotong University School of Medicine Yunye Liu

Department of Infectious Disease, Ruijin Hospital, Shanghai Jiaotong University School of Medicine Qing Xie

Department of Infectious Diseases, Ruijin Hospital, Shanghai Jiaotong University School of Medicine

\section{Wei Cai}

Department of Infectious Diseases, Ruijin Hospital, Shanghai Jiaotong University School of Medicine

\section{Shisan Bao}

Discipline of Pathology, School of Medical Sciences, Charles Perkin Centre, Faculty of Medicine and Health, The University of Sydney

Hui Wang ( $\nabla$ wanghuirj2@163.com )

\section{Research}

Keywords: Hepatitis B virus, HBV related HCC, IL 34, MCSF, therapeutic target

Posted Date: September 9th, 2020

DOI: https://doi.org/10.21203/rs.3.rs-68883/v1

License: (1) (1) This work is licensed under a Creative Commons Attribution 4.0 International License. Read Full License 


\section{Abstract}

Backgrounds: IL-34 is involved in a number of auto-immunities and cancers. We explored the relationship between serum/hepatic IL-34 and hepatitis B virus (HBV) related hepatocellular carcinoma (HBV-HCC) patients.

Methods: Serum was obtained from the HBV patients or healthy control with written consents. Liver tissue was obtained from liver biopsy in $\mathrm{CHB}, \mathrm{HBV}$ related cirrhosis patients or curative resection in HBV-HCC patients. Serum IL-34 and MCSF were measured, using ELISA. HepaticIL-34, MCSF and CD68 were determined, using immunohistochemistry.

Results: Serum IL-34 was 1.7, 1.6 or 1.7 fold higher in HBV-HCC than that of the other three groups (CHB, HBV related cirrhosis, and healthy control). Serum IL-34 was significantly reduced after trans-hepatic arterial chemoembolization (TACE) in HBV-HCC patients. There was significant correlation between the incidence of HBV-HCC and IL-34 $\left(r_{s}=0.160, p<0.05\right)$ as well as AFP $\left(r_{s}=0.442, p<0.01\right)$. Furthermore, intrahepatic IL-34 was higher in HBV-HCC than that of the other three groups. Intra-hepatic IL-34 was associated with high HBV-DNA, HBeAg', low tumor differentiation and small tumor size of HBV-HCC patients. Intra-hepaticCD68 ${ }^{+}$TAMs were increased 1.6 or 1.3 fold in HBV-HCC compared to that from $\mathrm{CHB}$ or HBV-cirrhosis. Intra-hepatic CD $68^{+}$TAMs were associated with high HBV-DNA, high tumor differentiation, big tumor size, abnormal AFP and more tumor number.

Conclusions: IL-34, correlated with HBV-HCC and IL-34, may be used as a therapeutic target in precise medicine for management of HBV-HCC.

\section{Introduction}

Hepatitis B virus (HBV) infection, a major health problem worldwide (1), is one of the major causes of hepatocellular carcinoma (HCC)(2). HBV related HCC (HBVHCC) is a common primary liver cancer with high mortality, high recurrence and low post-operative survival rate, mainly is due to later diagnosis. It is well known that host immunity plays a critical role in the carcinogenesis, which is elegantly demonstrated by the Nobel laureates for Medicine in 2018 (3).

Tumor microenvironment, including tumor cells, activate macrophages, cytokines and blood vessels, has an important role on the occurrence and development of tumor $(4,5)$. Tumor associated macrophage (TAMs) regulate the microenvironment $(6)$, but the role of TAMs is controversial $(7,8)$. TAMs promotes tumor invasion, formation of blood vessels and lymphatic vessel, migration of tumor cells $(9,10)$, perhaps via enhancing immunosuppression environment (11). In contrast, TAM may be involved in inhibiting cancer growing and metastasis (12). The explanation of such discrepancy might be due to differential polarization of macrophages during their maturation, namely macrophage 1 (M1) and M2 (12-15). The differential polarization of macrophages perhaps due to different microenvironments in different regions and/or in different individuals, mediated by different regulators $(14,16)$. 
IL34, a member of interleukin 1 family, is produced by a wide range of cells, including macrophages, fibroblasts and hepatocytes (17-19). IL34 promotes differentiation, proliferation and survival of mononuclear cells via binding to CSF1R (20). Dysregulation of IL34 is involved in many diseases(21), including inflammatory bowel disease (22), rheumatoid arthritis (23), chronic heart failure (24) and ischemia/reperfusion injuryincited acute kidney injury $(25,26)$. Macrophage colony stimulating factor (MCSF) [known as (colony stimulating factor1, CSF1)] is responsible for the survival, proliferation and differentiation of mononuclear phagocytes through biding to MCSFR (MCSF receptor; also known as CFS1R) (27). Although IL34 shares no apparent sequence homology with MCSF, the biological activity of IL34 is mediated via interacting with MCSFR, which is mainly expressed on the surface of macrophages (20).

Higher circulating IL34 and MCSF are detected in hepatitis C virus (HCV) patients with high fibrosis scores than that from the low scores or healthy subjects (19). Furthermore, serum IL34 may be associated with inflammatory activity and liver fibrosis in chronic hepatitis $B(\mathrm{CHB})(28)$. There is a close correlation between the severity of hepatic fibrosis and the incidence of HCC from both chronic hepatitis $\mathrm{B}$ and $\mathrm{C}$ patients (29). It is unclear the relationship between IL34 and the clinical presentation of HBVHCC, as well as the association between IL34 and TAM/MCSF. Understand the precise underlying mechanism of IL34 in regulating macrophage mediated tumorogenesis in HBVHCC, it could contribute to development of therapeutic agents, particularly in precision medicine.

\section{Results}

The baseline characteristics of the patients were summarized (Table 1). There were HBVHCC $(n=111)$, CHB $(n=64)$, HBVcirrhosis $(n=64)$ and HCs $(n=20)$, according to the inclusion criteria. 
Table 1

Demographic, clinical characteristic, biochemical characteristic

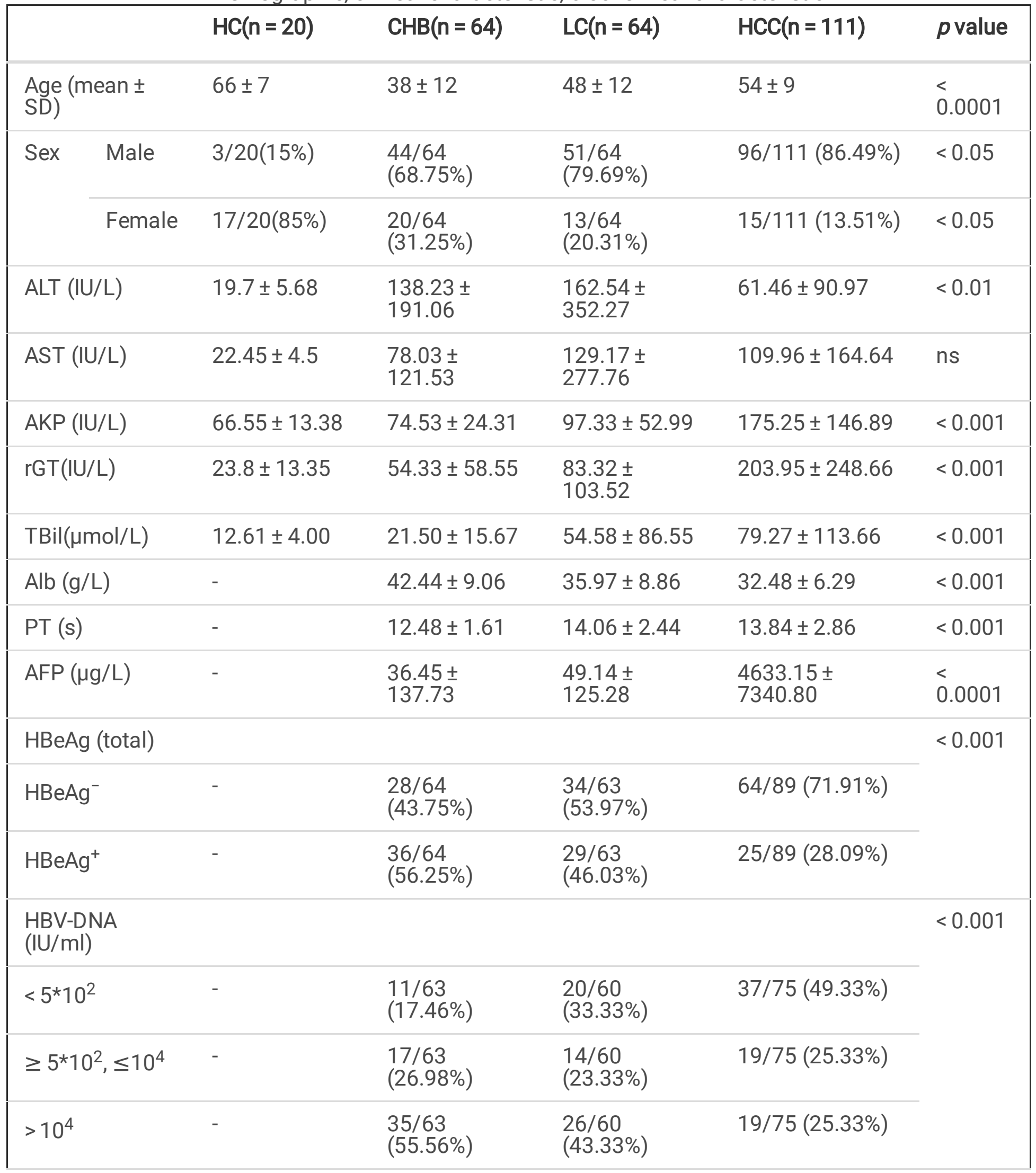

ALT: alanine aminotransferase; AST: aspartate aminotransferase; AKP: alklinephosphatase; rGT: gamma-glutamyl transpeptidase; TBil: total bilirubin; Alb: albumin; PT: prothrombin time; BCLC: Barcelona Clinic Liver Cancer; LC: liver cirrhosis. 


\begin{tabular}{|c|c|c|c|c|c|c|}
\hline & & $\mathrm{HC}(n=20)$ & $\mathrm{CHB}(n=64)$ & $\operatorname{LC}(n=64)$ & $\operatorname{HCC}(n=111)$ & $p$ value \\
\hline \multirow[t]{4}{*}{ BCLC } & $A$ & - & - & - & $17 / 111(15.32 \%)$ & - \\
\hline & $B$ & - & - & - & $30 / 111(27.03 \%)$ & - \\
\hline & $\mathrm{C}$ & - & - & - & $54 / 111(48.65 \%)$ & - \\
\hline & $\mathrm{D}$ & - & - & - & $10 / 111(9.01 \%)$ & - \\
\hline \multicolumn{2}{|c|}{ IL-34 (pg/ml) } & $21.51 \pm 11.86$ & $22.75 \pm 9.18$ & $26.58 \pm 15.83$ & $38.20 \pm 50.62$ & $<0.05$ \\
\hline \multicolumn{2}{|c|}{$\operatorname{MCSF}(p g / m l)$} & $\begin{array}{l}161.14 \pm \\
146.32\end{array}$ & $\begin{array}{l}134.66 \pm \\
138.68\end{array}$ & $\begin{array}{l}119.66 \pm \\
78.98\end{array}$ & $112.86 \pm 208.14$ & ns \\
\hline
\end{tabular}

\section{Serum IL34 was elevated in HBV-HCC, but not MCSF}

The levels of ALT, AST, AKP, rGT and TBil (reflecting liver function) were significantly higher in HBVHCC than CHB, HBVcirrhosis and HCs (all of $p<0.01$ ). AFP level was $36.45,49.14$, or $4633.15 \mu \mathrm{g} / \mathrm{l}$ from CHB, HBVcirrhosis, HBVHCC patients, respectively. Serum IL34, but not MCSF, was elevated in HBVHCC.

Serum IL34 was 1.7 or 1.6 fold higher from HBVHCC groups than that from CHB (38.20 vs $22.75, p<$ $0.05)$, HBVcirrhosis $(38.20$ vs $26.58, p<0.05)$ or HCs ( 38.20 vs $21.51, p<0.01)$ (Table 1 , Fig. $1 \mathrm{~A})$. There was no significant difference of IL34 between HBVcirrhosis patients and HCs (26.58 vs 21.51, $p>0.05$ ), nor between $\mathrm{CHB}$ patients and $\mathrm{HCs}(22.75$ vs $21.51, p>0.05)$. There was no significant difference of serum MCSF among HCs, CHB, HBVcirrhosis and HBVHCC groups (Table 1, Fig. 1B).

\section{Serum IL34 or MCSF during anti-tumor treatment in HBVHCC}

We further determined the serum IL34 or MCSF level in HBV-HCC patients who received TACE or surgery. Serum IL34 was decreased by $>20 \%$ post TACE in HBVHCC patients, compared to that prior to the treatment (24.12 vs $19.12, p<0.01$ ) (Fig. $2 \mathrm{~A})$; whereas no significant difference of MCSF was observed in the HBVHCC patients prior to and post TACE treatment (113.0 vs $112.9 ; 83.96$ vs $73.16 ; p>0.05$ ) (Fig. 2 C). In addition, in HBVHCC patients undergoing surgery, there was no significant change of circulating IL34 (13.56 vs $15.19, p>0.05$ ) (Fig. 2B) and MCSF (83.96 vs 73.16; $p>0.05$ ) (Fig. 2D) prior to and post-surgery.

\section{The factors thatare associated with HBV-HCC incidence}

If there is any correlation between IL-34 or MCSF and incidence of HBVHCC, as well as other HBV related influence factor, including ALT, AST, HBVDNA, HBsAg, HBeAg, AFP, using logistic regressive (Table 2). IL34 was positively correlated with the incidence of HBVHCC $\left(r_{s}=0.160, p<0.05\right)$, as well as, AFP $\left(r_{s}=0.442, p\right.$ 
< 0.01). HBsAg, HBeAg or HBVDNA were inversely correlated with HBVHCC $\left(r_{s}=-0.451, p<0.01 ; r_{s}=\right.$ $-0.295, p<0.01$; or $r_{s}=-0.322, p<0.01$, respectively). There was no correlation between circulating MCSF and the occurrence of HBV-HCC $(p>0.05)$.

Table 2

Factors associated with the incidence of HBVHCC

\begin{tabular}{|lll|}
\hline & \multicolumn{2}{c}{ HBVHCC } \\
\hline & $r_{s}$ & $p$ value \\
\hline $\mathrm{IL}-34(\mathrm{pg} / \mathrm{ml})$ & 0.160 & $<0.05$ \\
\hline $\mathrm{MCSF}(\mathrm{pg} / \mathrm{ml})$ & -0.127 & $<0.05$ \\
\hline ALT $(\mathrm{IU} / \mathrm{L})$ & -0.179 & $<0.01$ \\
\hline AST $(\mathrm{IU} / \mathrm{L})$ & 0.179 & $<0.01$ \\
\hline AKP $(\mathrm{IU} / \mathrm{L})$ & 0.410 & $<0.01$ \\
\hline $\mathrm{rGT}(\mathrm{IU} / \mathrm{L})$ & 0.402 & $<0.01$ \\
\hline TBil $(\mu \mathrm{mol} / \mathrm{L})$ & 0.298 & $<0.01$ \\
\hline Alb $(\mathrm{g} / \mathrm{L})$ & -0.411 & $<0.01$ \\
\hline PT $(\mathrm{s})$ & 0.109 & $\mathrm{~ns}$ \\
\hline AFP $(\mu \mathrm{g} / \mathrm{L})$ & 0.442 & $<0.01$ \\
\hline HBsAg & -0.451 & $<0.01$ \\
\hline HBeAg & -0.295 & $<0.01$ \\
\hline HBV-DNA $(\mathrm{IU} / \mathrm{ml})$ & -0.322 & $<0.01$ \\
\hline ALT: & & \\
\hline
\end{tabular}

ALT: alanine aminotransferase; AST: aspartate aminotransferase; AKP: alkline phosphatase; rGT: gamma-glutamyl transpeptidase; TBil: total bilirubin; Alb: albumin; PT: prothrombin time.

\section{Intrahepatic IL34 from CHB, HBVcirrhosis, HBVHCC patients}

According to the inclusion criteria, we obtained specimens of liver tissue from $\operatorname{HBVHCC}(n=30), \mathrm{CHB}$ patients $(n=5), H B V$ cirrhosis $(n=5), H C s(n=5)$. There was no significantly difference of biochemical indices (ALT, AST, AKP, GGT, TBil, PT and AFP) among CHB, HBVcirrhosis, and HBVHCC patients, except Alb (Table 4).IntrahepaticlL34 from HBVHCC patients was significantly higher than that of CHB, HBVcirrhosis and HCs (Fig. 3, 4)(all of the $p<0.05$ ). Intrahepatic MCSF and CD68 ${ }^{+}$TAMs were increased $2.2,4.4,1.6$ or 1.3 fold in HBVHCC, compared to that from CHB or HBVcirrhosis, respectively (Fig. 4). Interestingly, intrahepatic MCSF and $\mathrm{CD}^{+} 8^{+}$TAMs were significantly lower than $\mathrm{HCs}(p<0.05)$. 
Table 4

Baseline characteristics of the study population of immunohistochemistry

\begin{tabular}{|c|c|c|c|c|c|}
\hline & & $\mathrm{CHB}(\mathrm{n}=5)$ & $L C(n=5)$ & $\operatorname{HCC}(n=30)$ & $p$ value \\
\hline Age(mean : & SD) & $35.40 \pm 5.41$ & $49.20 \pm 6.46$ & $59.43 \pm 14.71$ & $<0.01$ \\
\hline Sex & Male & $4 / 5(80 \%)$ & $3 / 5(60 \%)$ & $26 / 30(86.67 \%)$ & $<0.05$ \\
\hline & Female & $1 / 5(20 \%)$ & $2 / 5(40 \%)$ & 4/30(13.33\%) & $<0.05$ \\
\hline ALT (IU/L) & & $69.00 \pm 64.82$ & $73.20 \pm 60.32$ & $44.67 \pm 55.48$ & ns \\
\hline AST (IU/L) & & $43.50 \pm 33.13$ & $47.80 \pm 33.21$ & $208.33 \pm 927.59$ & ns \\
\hline AKP (IU/L) & & $64.00 \pm 26.67$ & $68.60 \pm 8.91$ & $76.03 \pm 35.93$ & ns \\
\hline rGT (IU/L) & & $31.50 \pm 21.38$ & $51.60 \pm 37.10$ & $55.40 \pm 45.34$ & ns \\
\hline TBil $(\mu \mathrm{mol} / \mathrm{l}$ & & $15.05 \pm 3.96$ & $10.58 \pm 5.40$ & $16.68 \pm 8.96$ & ns \\
\hline Alb $(g / L)$ & & $43.50 \pm 3.51$ & $44.40 \pm 1.14$ & $36.77 \pm 6.73$ & $<0.05$ \\
\hline PT (s) & & $12.05 \pm 1.42$ & $12.06 \pm 0.83$ & $11.87 \pm 0.91$ & ns \\
\hline $\operatorname{AFP}(\mu \mathrm{g} / \mathrm{L})$ & & $4.95 \pm 3.42$ & $15.38 \pm 22.77$ & $1577.71 \pm 3349.61$ & ns \\
\hline HBV-DNA & $<5 * 10^{2}$ & $1 / 5(20 \%)$ & $3 / 5(60 \%)$ & $16 / 26(61.5 \%)$ & ns \\
\hline$(\mathrm{IU} / \mathrm{ml})$ & $\geq 5^{*} 10^{2}$ & $4 / 5(80 \%)$ & $2 / 5(40 \%)$ & $10 / 26(38.5 \%)$ & \\
\hline
\end{tabular}

\section{Correlation between intrahepatic IL34, MCSF and CD68 + TAMs in HBVHCC and clinical parameters}

Associations between clinical pathological parameters of HBVHCC and IL34, MCSF or CD68 ${ }^{+}$TAMs were listed (Table 3). IL34 was associated with HBVDNA, HBeAg, tumor differentiation and tumor size of HBVHCC patients (Fig. 5). IL34 was $28 \%$ lower in the group of patients with low HBVDNA level compared to patients with high level $(p<0.05)$. Nearly $50 \%$ reduced intra-hepatic IL34 was also observed in $\mathrm{HBeAg}^{+}$ compared to $\mathrm{HBeAg}^{-} \mathrm{HBVHCC}$ patients $(p<0.05)$. In addition, there was a significant inverse correlation between IL-34 and differentiation or tumor size of HCC. IL34 was $37 \%$ increased in low differentiated HCC compared to that of high differentiation group $(p<0.05)$; as well as, $\sim 36 \%$ intrahepatic IL-34 production from small tumor size $(\leq 5 \mathrm{~cm})$ than that from big size tumor group $(p<0.05)$. However, there was no correlation between IL-34 and other parameters, including tumor number and AFP of HCC. Intrahepatic CD68 ${ }^{+}$TAMs were associated with high HBVDNA, high tumor differentiation, big tumor size, abnormal AFP and more tumor number. MCSF was associated with HBVDNA, HBeAg, AFP, tumor number of HBVHCC patients, except tumor differentiation and tumor size. 
Table 3

Correlations between intrahepatic IL34, MCSF and CD68 ${ }^{+}$TAMs and clinical features in patients with $\operatorname{HBVHCC}(\mathrm{n}=30)$

\begin{tabular}{|c|c|c|c|c|c|c|c|c|}
\hline \multirow[t]{2}{*}{ Characteristics } & & \multirow[t]{2}{*}{$\mathrm{N}$} & \multicolumn{2}{|l|}{ IL-34 } & \multicolumn{2}{|l|}{ MCSF } & \multicolumn{2}{|c|}{ CD $68^{+}$TAMs } \\
\hline & & & Median & $p$ value & Median & $p$ value & Median & $p$ value \\
\hline \multirow{2}{*}{$\begin{array}{l}\text { HBV-DNA } \\
(\mathrm{IU} / \mathrm{mL})\end{array}$} & $<5^{\star} 10^{2}$ & 15 & 35.7 & $<0.001$ & 122.3 & $<0.01$ & 21.5 & $<0.05$ \\
\hline & $\geq 5^{\star} 10^{2}$ & 11 & 49.4 & & 56.3 & & 26.3 & \\
\hline \multirow[t]{2}{*}{ HBeAg } & $\mathrm{HBeAg}^{-}$ & 10 & 45.3 & $<0.01$ & 134.7 & $<0.05$ & 25.8 & ns \\
\hline & $\mathrm{HBeAg}^{+}$ & 3 & 24.6 & & 50.0 & & 23.0 & \\
\hline \multirow[t]{2}{*}{ AFP } & normal & 14 & 44.4 & ns & 57.2 & $<0.05$ & 19.2 & $<0.0001$ \\
\hline & abnormal & 16 & 43.0 & & 101.2 & & 27.2 & \\
\hline \multirow[t]{2}{*}{ Differentiation } & $\leq$ if & 10 & 54.9 & $<0.05$ & 90.3 & ns & 16.5 & $<0.0001$ \\
\hline & $>11$ & 19 & 34.8 & & 75.1 & & 26.1 & \\
\hline \multirow[t]{2}{*}{ Tumor number } & 1 & 22 & 43.4 & ns & 89.1 & $<0.05$ & 20.1 & $<0.0001$ \\
\hline & $\geq 0$ & 7 & 39.1 & & 46.0 & & 31.7 & \\
\hline \multirow[t]{2}{*}{ Tumor size } & $\leq \mathrm{u}$ & 12 & 53.9 & $<0.0001$ & 66.0 & ns & 25.0 & $<0.001$ \\
\hline & $>5$ & 17 & 34.8 & & 88.1 & & 21.0 & \\
\hline
\end{tabular}

\section{Discussion}

In the present study, we evaluated circulating and intra-hepatic IL34 in HBV related liver diseases. Serum IL34 from HBVHCC patients was significantly higher than that of CHB, HBVcirrhosis and HCs. IL34 and AFP were correlated with the incidence of HBVHCC. Furthermore, serum IL34 was decreased following antitumor TACE treatment in HBVHCC. Intrahepatic IL34 from these groups was consistent with serum IL34. Intrahepatic IL34 was associated with high HBVDNA, $\mathrm{HBeAg}^{-}$, low tumor differentiation and small tumor size of HBV-HCC patients. Intrahepatic CD68 ${ }^{+}$TAMs were increased in HBVHCC compared to that from $\mathrm{CHB}$ and HBVcirrhosis. Intrahepatic $\mathrm{CD} 68^{+}$TAMs were associated with high HBVDNA, high tumor differentiation, big tumor size, abnormal AFP and more tumor number.

Chronic HBV infection is a major risk factor for HCC globally, which is supported by the findings, showing a close correlation among chronic viral hepatitis, liver cirrhosis after hepatitis, primary liver cancer (29). It is well documented there is close association between HBV infection, as well as, the level of HBsAg, and 
the incidence of $\operatorname{HCC}(30,31)$. Based on the APSAL guideline, it is considered that CHB, liver cirrhosis, $\mathrm{HCC}$ are reflecting the different stages of the liver diseases. Up to date, two major therapeutic strategies are applied for $\mathrm{CHB}$, i.e. direct acting antiviral drugs and immune modulatory agents (32). However, there is no effective treatment to cure HBV infection, resulting in certain $\mathrm{CHB}$ patients progress to cirrhosis and HCC (1). Therefore, early detection, diagnosis and treatment are the key points in reducing the incidence of HBVHCC.

In our current study, serum IL34 from HBVHCC patients was significantly higher than that of CHB, HBV cirrhosis, and HCs, suggesting that IL34 may contribute to tumorigenesis of HCC, enhancing progression from CHB patients to cirrhosis and finally HCC. Furthermore, intra-hepatic IL34 was consistent with the result of serum. Our results are in line with previous studies documenting high IL34 in pathological conditions $(22,23,33)$. IL34 is overexpressed in the inflamed synovium of patients with rheumatoid arthritis, where it perhaps acting synergistic with TNF and IL $1 \beta$, induces osteoclastogenesis and contributes to tissue inflammation and bone erosion (34).

The development of tumor is closely related to the microenvironment, including tumor cells, monocytes/macrophages, cytokines and neovasculization. TAMs are mixed phenotype, expressing M1 or M2 markers (13), and may be influenced by different microenvironments in different regions and/or in different individuals. CD68, regardless the status of their polarization in many studies, has been widely used as a marker in marking TAMs which is infiltrating in solid tumors (35). In our current study, intrahepatic $\mathrm{CD} 68^{+}$TAMs were increased gradually with the order from $\mathrm{CHB}, \mathrm{HBV}$ cirrhosis to HBVHCC patients. Our current observation invites speculation that the increased infiltrating CD $68^{+}$TAMs may be M1 dominant, contributing to antitumor activity, which will be determined in our future experiments.

It was reported that IL34 was upregulated in hepatitis C virus infection and inhibited the production of IFNY(19), and IL34 may also be associated with inflammatory activity and liver fibrosis in CHB (28). Moreover, baseline serum IL34 levels seem to serve as a prognostic factor for progression in such patients. In our current study, there was only IL34 significantly decreased in postantitumor treatment compared to that of pre-treatment of HBVHCC in serum. Serum IL34 was significantly correlated with the incidence of HBVHCC. This further suggests that IL34 could be used as a predictor for HBVHCC, which will be further determined in vitro and in vivo.

IL34 induces differentiation of leukemia cells into monocytelike, macrophagelike cells and mature macrophages through the JAK/STAT and PI3K/Akt signaling pathways (36), suggesting IL34 enhances differentiation of other cancers $(15,21)$. This is consistent with our current finding that IL34 was correlated with the differentiation and tumor size of HBVHCC. In addition, intrahepatic IL34 was associated with HBVDNA, HBeAg, tumor differentiation and tumor size of HBVHCC patients. Our data may provide an explanation for the possible role of IL34 in the development of HBVHCC, i.e. IL34 also regulates HBVHCC differentiation, which would have potential clinical relevance regarding IL34 as a therapeutic target for malignancy. IL-34 is able to inhibit HBV replication in vivo and in vitro (37). Such 
finding is in line with our current clinical study, showing that IL-34 is beneficially to the HCC patients for potential therapeutic target.

We acknowledge that there are limitations in the current study. There is no kinetics of intrahepatic or circulating IL34 during the development and management of HCC, and no correlation between IL34 and prognosis is detected. These two interesting points will be determined in our future study. We also acknowledge that the sample size in the current study is relatively small, which may not perfectly reflect the real cases. However the current experiment is just a proof of concept. We will explore the underlying mechanism with large sample size and multiple center studies in future.

In summary, the current study improves our understanding of the role of IL34 in HBV related liver disease. Increased IL34 may contribute to the transformation of the tumor, which is a potential predictor of HBVHCC. The underlying mechanism of IL34 in HBVHCC is being currently investigated.

\section{Materials And Methods}

\section{Study population}

All of the patients were identified between April 2015 and July 2017 in Department of Infectious Diseases, Shanghai Ruijin Hospital. Serum and liver tissues were obtained from the patients with informed consents. It was selected as healthy controls (HCs) that age and sex matched healthy people for routine health check in our hospital without liver disease/HBsAg negative/negative image in CT or MRI. The selection of treatment for HBV-HCC patients were based on the guideline for treatment of primary liver cancer in China, and was conducted with a multidisciplinary diagnosis and treatment team of Ruijin Hospital, as described previously(38). HBV-HCC patients selected for the current study were received eithertranshepatic arterial chemoembolization (TACE) or curative resection treatment. TACE, an interventional treatment, is one of the most common nonsurgical treatments for liver cancer (38). Curative resection is a surgical procedure that hepatocellular cancerous tissue and a certain amount of normal tissue to be removed to obtain adequate margins. The purpose is to minimize the risk of any cancer cells being left behind(39).

The inclusion criteria of $\mathrm{CHB}$ patients were: first, adult with consecutive $\mathrm{HBsAg}^{+}$for at least six months, nucleos(t)ide (NA)naïve without cirrhosis or carcinoma.

Second,1) Adult with consecutive $\mathrm{HBsAg}^{+}$for at least six months; 2) Diagnosed as primary HCC confirmed with pathology; 3 ) alpha-fetoprotein (AFP) $>400 \mu \mathrm{g} / \mathrm{L}$, no other active liver disease, pregnancy, embryonic source sex reproductive system tumor and metastatic liver cancer, and could touch a swelling or hard of the liver with tumor, or imaging examination, such as computerized tomography (CT)(40), magnetic resonance imaging (MRI) scans (41), and ultrasound examinations, revealed liver occupying lesions characteristic; 4) AFP $\leq 400 \mu \mathrm{g} / \mathrm{L}$, more than two imaging examinations revealed liver occupying lesions which has characteristic of HCC. 
Third, 1) adult with consecutive $\mathrm{HBsAg}^{+}$for at least six months; 2 ) diagnosed cirrhosis by biopsy of the liver; 3 ) or imaging examination, such as $\mathrm{CT}, \mathrm{MRI}$, FibroScan or ultrasound examinations, detected enlarged livers, abnormally nodular livers, enlarged spleens, and fluid in the abdomen, suggesting cirrhosis; 4) the hospitalized patients under any event can also be diagnosed as decompensation liver cirrhosis: abdominal cavity effusion, esophageal gastric varices burst out of the blood, hepatic encephalopathy, infection.

The exclusion criteria included: 1) Coinfected with HIV, HAV, HCV, HDV or HEV; 2) Undergone liver transplantation before the study; 3 ) Autoimmune liver disease, nonalcoholic fatty liver disease, alcoholic fatty liver disease, Wilson's disease, or hemochromatosis; 4) Pregnant women or breast-feeding; 5) Liver metastatic tumors; 6) CHB related acuteon-chronic liver failure (ACLF).

The exclusion criteria of healthy people were: 1) Undergone liver disease before the study; 2) Had abnormal liver function recently; 3 ) alcoholism (amount of alcohol: female $\geq 20 \mathrm{~g} / \mathrm{d}$, male $\geq 30 \mathrm{~g} / \mathrm{d}$ ).

This study complies with the declaration of Helsinki, and the study protocol was approved by the Human Ethics Committee, Ruijin Hospital. Written informed consent was obtained from all of the patients according to standards of the local ethics committees.

\section{Routine biochemistry and cytokine quantification}

It was performed that routine biochemistry [alanine aminotransferase (ALT), aspartate aminotransferase (AST), alkaline phosphatase (AKP), gamma-glutamyl transpeptidase ( $\mathrm{rGT}$ ), total bilirubin (TBil), albumin (Alb) and prothrombin time (PT)] and virologic tests [HBVDNA level, hepatitis B surface antigen (HBsAg), hepatitis $B$ surface antibody (antiHBs), hepatitis $B$ envelop antigen ( $\mathrm{HBeAg}$ ), hepatitis B envelop antibody (antiHBe)]. $\mathrm{HBeAg}^{+}$is defined as positive hepatitis $\mathrm{B}$ envelop antigen, and $\mathrm{HBeAg}^{-}$is defined as negative hepatitis B envelop antigen. The Scheuer's scoring system was applied for pathology diagnosis of inflammation and fibrosis grading of liver tissue (42). Liver cirrhosis is defined as $\geq S 4$ of Scheuer's scoring system. Serum ALT, AST, AKP, rGT, TBil, and Alb (reflecting liver function) were quantified using Beckman coulter AU5800 automatic biochemical analyzer. HBsAg, anti-HBs, HBeAg and anti-HBe were determined using commercial ELISA kits (Abbott Diagnostics, IL). Serum HBV DNA levels were measured using qPCR, Roche Amplicor (Roche Diagnostic Systems, Branchburg, NJ, USA). Serum IL34 and MCSF were quantified using ELISA (R\&D Systems, Lille, France). Results were expressed as a concentration of cytokine production.

\section{Immunohistochemistry (IHC)}

The liver tissue blocks were obtained from surgery for HBVHCC $(n=30)$ or from liver biopsy for diagnosis of CHB liver $(n=5)$ and HBV-cirrhosis liver $(n=5)$ or the off cuts from liver transport donors for HCs $(n=$ 5). HCs did not present liver disease/ HBsAg negative/negative image in CT or MRI. Hepatic IL-34, MCSF and CD68 were determined using immunohistochemistry, using 3, 3'diaminobenzidine (DAB) color development, as described previously (43). The primary antibodies were polyclonal rabbit antihuman IL34 (bs-18170R, Beijing Biosynthesis Biotechnology, China), polyclonal rabbit anti-human MCSF (Abcam, 
Cambridge, UK) and monoclonal mouse antihuman CD68 (Dako, Copenhagen, Denmark). The secondary antibody (Beijing Sequoia Jinqiao Biological Technology) was used. The specific target(s) was visualized with DAB detection kit and counterstained with hematoxylin. The IHC was repeated for twice. Negative control was applied in each labeling for every primary rabbit negative control. Intra-hepaticlL-34 or MCSF is localized in the cytoplasma of hepatocytes, which has been well documented in our previous publications (44). IHC was quantified using a computer-assisted genuine color image analysis system (ImageProplus 9.0) for hepatic IL34, MCSF or CD68, as described previously $(44,45)$.

\section{Statistical analysis}

Continuous variables were expressed as means \pm standard deviation or median (inter-quartile range) where appropriate. Differences between two groups were determined by unpaired $t$ test or the MannWhitney $U$ test. Among three groups were used by analysis of variance (ANOVA) or the KruskalWallis $\mathrm{H}$ nonparametric test. Chisquare or Fisher's exact test was employed to compare nominal variables. Figure 1, 2, 4, 5 were generated using GraphPad Prism, Version 8.0 (GraphPad Software, San Diego, CA, USA). All statistical tests are twoside, and $p$-value $<0.05$ was considered to be statistically significant. SPSS version 22.0 was used for all statistical analysis (SPSS Inc., Chicago, IL, USA).

\section{Abbreviations}

ACLF, acuteon-chronic liver failure; AFP alpha-fetoprotein; AKP:alkline phosphatase; ALT, alanine aminotransferase; Alb:albumin; ANOVA, analysis of variance; antiHBe, hepatitis B envelop antibody; antiHBs, hepatitis B surface antibody; AST, aspartate aminotransferase; $\mathrm{CHB}$, chronic hepatitis $\mathrm{B} ; \mathrm{CSF}-1$, colony stimulating factor $1 ; \mathrm{HAV}$, hepatitis A virus; HBV, hepatitis $B$ virus; $H B V H C C$, hepatitis $B$ virus related hepatocellular carcinoma; HBVcirrhosis, hepatitis $B$ virus related cirrhosis; $\mathrm{HBeAg}$, hepatitis $B$ envelop antigen; $\mathrm{HBsAg}$, hepatitis $B$ surface antigen; $\mathrm{HCC}$, hepatocellular carcinoma; HCs, healthy controls; $\mathrm{HCV}$, hepatitis $\mathrm{C}$ virus; $\mathrm{HDV}$, hepatitis D virus; HEV, hepatitis E virus; HIV, human immunodeficiency virus; MCSF, macrophage colony stimulating factor; MCSFR, macrophage colony stimulating factor receptor; PT:prothrombin time; rGT:gamma-glutamyl transpeptidase; TACE, transhepatic arterial chemoembolization; TAMs, tumor associated macrophage; TBil:total bilirubin.

\section{Declarations}

\section{Conflict of Interest:}

The authors declare that there is no conflict of interest.

\section{Authors' contributions:}


H.W., C.W. and S.B. designed the research. K.H.L., Y.W. and Q.X. conducted the research. H.W. provided financial support for the study. K.H.L., Y.Z.D., Q.Q.Z., L.Y, J.D.X. and Y.Y.L. collected the data of patients. All authors prepared the figures, tables and read and approved the manuscript.

\section{Acknowledgments:}

We appreciate the support from the staffs from the Department of Infectious Diseases, Ruijin Hospital.

Financial support statement: This work was supported by grants from the National Natural Science Foundation of China (81570560), The Shanghai key project of Integrated Traditional Chinese and Western Medicine (ZY (2018-2020)-FWTX-3001), Technology Supporting Project of the Science and Technology Commission Shanghai Municipality (16411960300), Shanghai Municipal Key Clinical Specialty(Infectious disease, YW20190002), The Suzhou Expert Team of Clinical Medicine (SZYJTD201717), National Science and Technology Major Project (2018ZX10205504-001-002, 2014ZX10005001), Shanghai Municipal Hospitals project: Improving Clinical Skills of Major Diseases (16CR1026B), and SJTU 2019, The Univeristy of Sydney (S.B.).

\section{References}

1. Ott JJ, Stevens GA, Groeger J, Wiersma ST. Global epidemiology of hepatitis B virus infection: new estimates of age-specific HBsAg seroprevalence and endemicity. Vaccine. 2012;30(Mar 9):2212.

2. F Bray et al. Global cancer statistics 2018: GLOBOCAN estimates of incidence and mortality worldwide for 36 cancers in 185 countries. Cancer J Clin. 2018;68(Nov):394.

3. Leach DR, Krummel MF, Allison JP. Enhancement of antitumor immunity by CTLA-4 blockade. Science. 1996;271(Mar 22):1734.

4. Spill F, Reynolds DS, Kamm RD, Zaman MH. Impact of the physical microenvironment on tumor progression and metastasis. Current opinion in biotechnology 40, 41 (Aug, 2016).

5. Joyce JA, Fearon DT. T cell exclusion, immune privilege, and the tumor microenvironment. Science. 2015;348(Apr 3):74.

6. Petty AJ, Yang Y. Tumor-associated macrophages: implications in cancer immunotherapy. Immunotherapy. 2017;9(Mar):289.

7. Zhu Y, et al. Tissue-Resident Macrophages in Pancreatic Ductal Adenocarcinoma Originate from Embryonic Hematopoiesis and Promote Tumor Progression. Immunity. 2017;47:323. (Aug 15.

8. MR Jadus et al., Human U251MG glioma cells expressing the membrane form of macrophage colony-stimulating factor (mM-CSF) are killed by human monocytes in vitro and are rejected within immunodeficient mice via paraptosis that is associated with increased expression of three different heat shock proteins. Cancer gene therapy 10, 411 (May, 2003).

9. Wu H, et al. Tumor-associated macrophages promote angiogenesis and lymphangiogenesis of gastric cancer. Journal of surgical oncology. Sep 15, 2012;106:462. 
10. Coussens LM, Werb Z. Inflammation and cancer. Nature. 2002;420:860. (Dec 19-26.

11. Biswas SK, Mantovani A. Macrophage plasticity and interaction with lymphocyte subsets: cancer as a paradigm. Nature immunology. 2010;11(Oct):889.

12. Wynn TA, Chawla A, Pollard JW. Macrophage biology in development, homeostasis and disease. Nature. 2013;496:445. (Apr 25.

13. ED Foucher et al. IL-34 induces the differentiation of human monocytes into immunosuppressive macrophages. antagonistic effects of GM-CSF and IFNgamma. PloS one. 2013;8:e56045.

14. Glass CK, Natoli G. Molecular control of activation and priming in macrophages. Nature immunology. 2016;17(Jan):26.

15. Bao S, Hu R, Hambly BD. IL-34, IL-36 and IL-38 in colorectal cancer-key immunoregulators of carcinogenesis. Biophys Rev, (Jul 7, 2020).

16. Al Segaliny et al. Interleukin-34 promotes tumor progression and metastatic process in osteosarcoma through induction of angiogenesis and macrophage recruitment. International journal of cancer. 2015;137(Jul 1):73.

17. $\mathrm{H}$ Lin et al. Discovery of a cytokine and its receptor by functional screening of the extracellular proteome. Science. 2008;320(May 9):807.

18. Greter $M$, et al. Stroma-derived interleukin-34 controls the development and maintenance of langerhans cells and the maintenance of microglia. Immunity. Dec 14, 2012;37:1050.

19. L Preisser et al. IL-34 and macrophage colony-stimulating factor are overexpressed in hepatitis $C$ virus fibrosis and induce profibrotic macrophages that promote collagen synthesis by hepatic stellate cells. Hepatology. 2014;60(Dec):1879.

20. Ma X, et al. Structural basis for the dual recognition of helical cytokines IL-34 and CSF-1 by CSF-1R. Structure. 2012;20(Apr 4):676.

21. Q Liu et al. Inverse correlation between Interleukin-34 and gastric cancer, a potential biomarker for prognosis. Cell Biosci. 2020;10:94.

22. S Zwicker et al. Interleukin 34: a new modulator of human and experimental inflammatory bowel disease. Clinical science. 2015;129(Aug):281.

23. SH Chang et al. Baseline serum interleukin-34 levels independently predict radiographic progression in patients with rheumatoid arthritis. Rheumatol Int. 2015;35(Jan):71.

24. Q Fan et al. IL-34 is associated with the presence and severity of renal dysfunction and coronary artery disease in patients with heart failure. Scientific reports. 2016;6(Dec 16):39324.

25. Edwards JK. Acute kidney injury: IL-34 promotes persistent ischaemia-induced AKI. Nat Rev Nephrol. 2015;11(Sep):504.

26. JH Baek et al. IL-34 mediates acute kidney injury and worsens subsequent chronic kidney disease. J Clin Investig. 2015;125(Aug 3):3198.

27. ER Stanley et al. Biology and action of colony-stimulating factor-1. Mol Reprod Dev. 1997;46(Jan):4. 
28. Wang YQ, Cao WJ, Gao YF, Ye J, Zou GZ, Serum interleukin-34 level can be an indicator of liver fibrosis in patients with chronic hepatitis B virus infection. World journal of gastroenterology 24, 1312 (Mar 28, 2018).

29. Maucort-Boulch D, de Martel C, Franceschi S, Plummer M. Fraction and incidence of liver cancer attributable to hepatitis B and C viruses worldwide. International journal of cancer. 2018;142(Jun 15):2471.

30. e. e. e. European Association for the Study of the Liver. Electronic address, L. European Association for the Study of the, EASL 2017 Clinical Practice Guidelines on the management of hepatitis B virus infection. Journal of hepatology 67, 370 (Aug, 2017).

31. TC Yip et al. HBsAg seroclearance further reduces hepatocellular carcinoma risk after complete viral suppression with nucleos(t)ide analogues. Journal of hepatology. 2019;70(Mar):361.

32. NA Terrault et al. Update on prevention, diagnosis, and treatment of chronic hepatitis B: AASLD 2018 hepatitis B guidance. Hepatology. 2018;67(Apr):1560.

33. Wang H, Cao J, Lai X. Serum Interleukin-34 Levels Are Elevated in Patients with Systemic Lupus Erythematosus. Molecules 22, (Dec 28, 2016).

34. Darrieutort-Laffite C, et al. IL-1beta and TNFalpha promote monocyte viability through the induction of GM-CSF expression by rheumatoid arthritis synovial fibroblasts. Mediat Inflamm. 2014;2014:241840.

35. AM Jubb et al., Expression of vascular notch ligand delta-like 4 and inflammatory markers in breast cancer. The American journal of pathology 176, 2019 (Apr, 2010).

36. Booker BE, Clark RS, Pellom ST, Adunyah SE. Interleukin-34 induces monocytic-like differentiation in leukemia cell lines. Int J Biochem Mol Biol. 2015;6:1.

37. ST Cheng et al. Interleukin-34 inhibits hepatitis B virus replication in vitro and in vivo. PloS one. 2017;12:e0179605.

38. J Zhou et al. Guidelines for Diagnosis and Treatment of Primary Liver Cancer in China (2017 Edition). Liver cancer. 2018;7(Sep):235.

39. EC Lai et al. Hepatic resection for hepatocellular carcinoma. An audit of 343 patients. Annals of surgery. 1995;221(Mar):291.

40. YJ Lee et al. Hepatocellular carcinoma: diagnostic performance of multidetector CT and MR imaginga systematic review and meta-analysis. Radiology. 2015;275(Apr):97.

41. Joo I, Lee JM. Recent Advances in the Imaging Diagnosis of Hepatocellular Carcinoma: Value of Gadoxetic Acid-Enhanced MRI. Liver cancer. 2016;5(Feb):67.

42. Scheuer PJ. Classification of chronic viral hepatitis: a need for reassessment. Journal of hepatology. 1991;13(Nov):372.

43. Xiang X, et al. IL-22 and non-ELR-CXC chemokine expression in chronic hepatitis B virus-infected liver. Immunol Cell Biol. 2012;90(Jul):611. 
44. R Lai et al. Protective effect of Th22 cells and intrahepatic IL-22 in drug induced hepatocellular injury. Journal of hepatology. 2015;63(Jul):148.

45. Chen F, et al. Interleukin-38 in colorectal cancer: a potential role in precision medicine. Cancer Immunol Immunother. 2020;69(Jan):69.

Figures

Figure 1

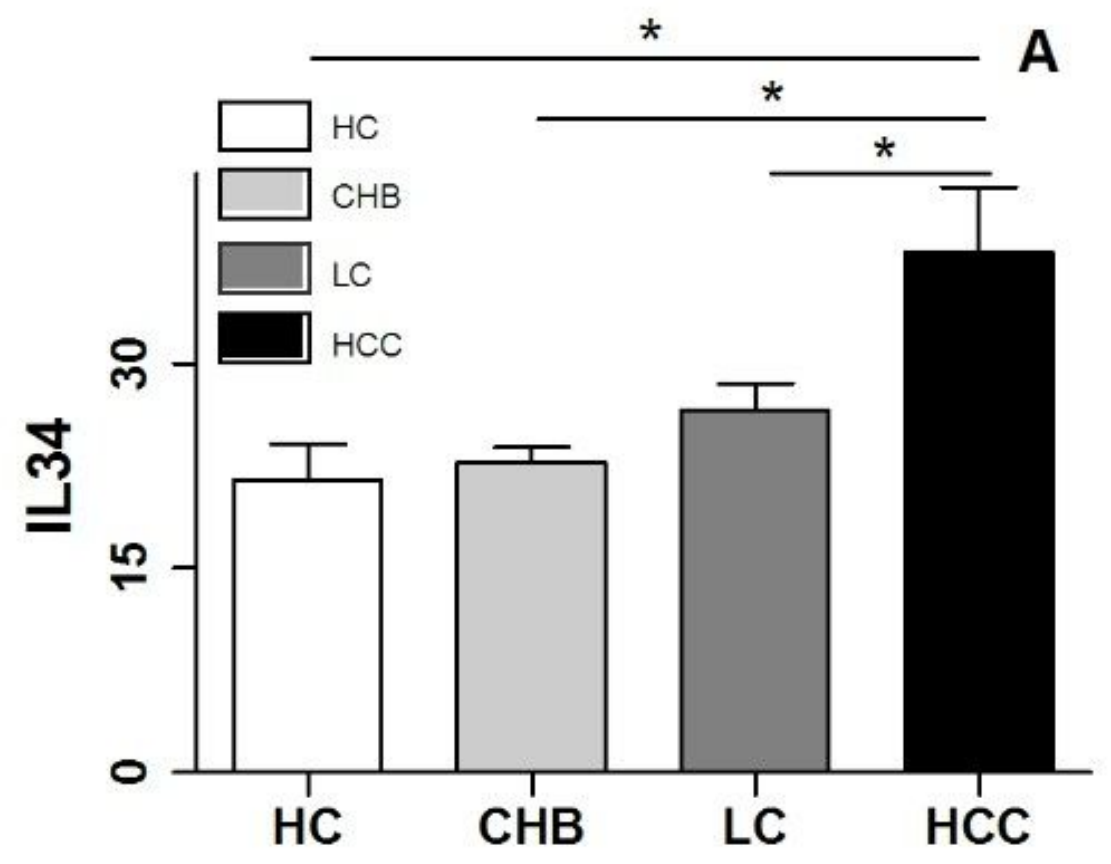

B

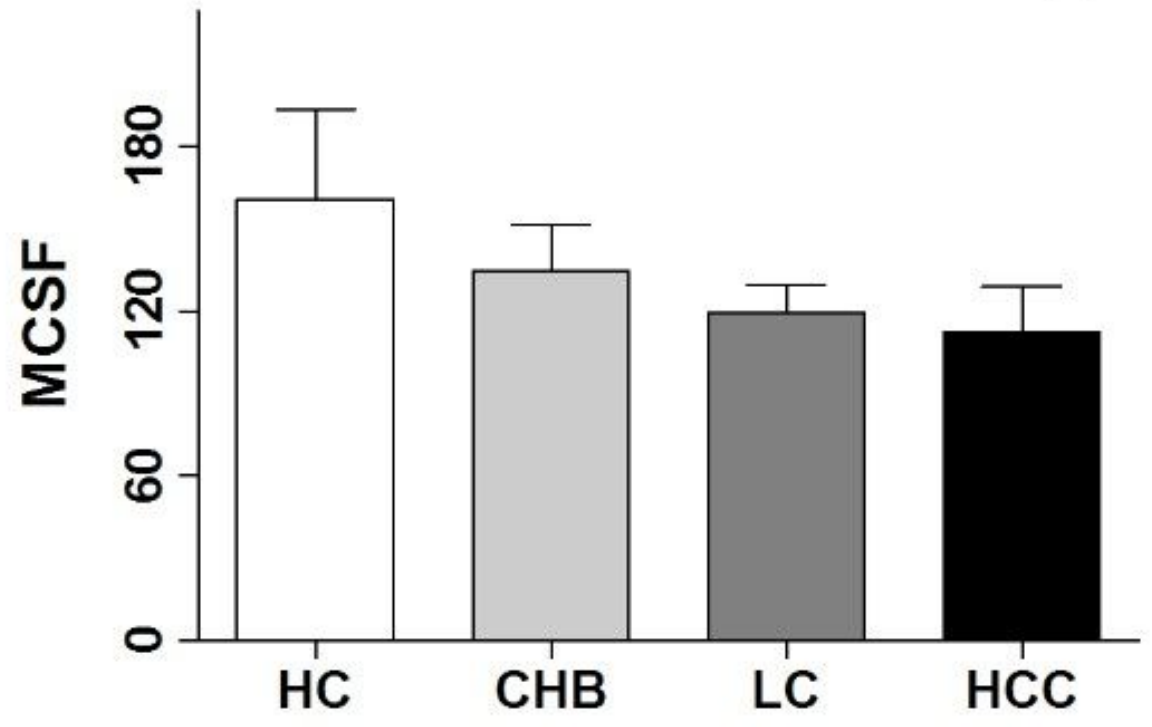


Figure 1

A. Serum IL 34 of HCs, CHB, HBV cirrhosis and HBV HCC; B. Serum MCSF of HCs, CHB, HBV cirrhosis and HBV HCC. (LC: liver cirrhosis)

\section{Figure 2}
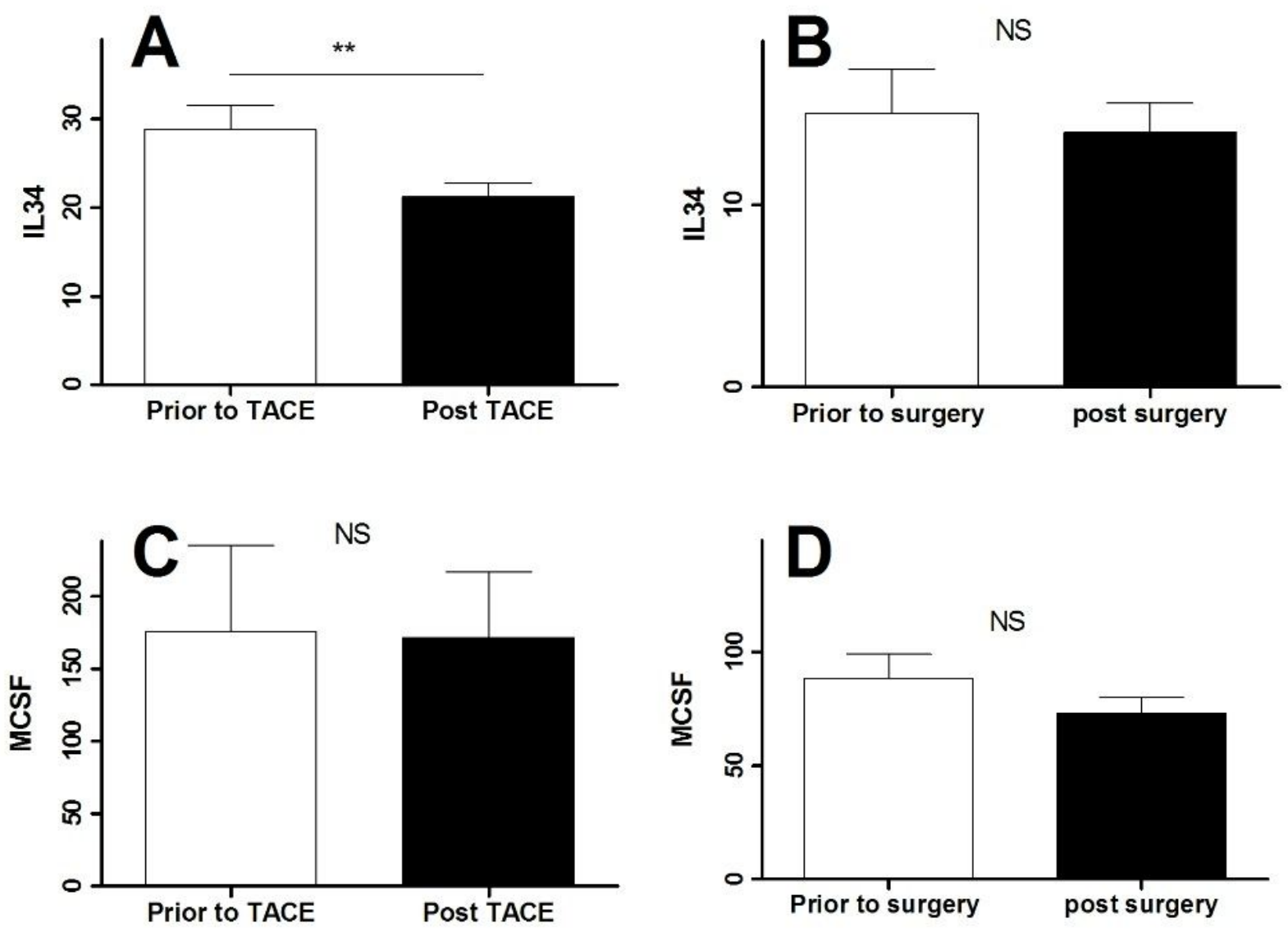

Figure 2

A. The serum IL 34 in HBV HCC patients prior to and post trans hepatic arterial chemotherapy and embolization (TACE); B. Serum IL 34 in HBV HCC patients prior to and post surgery; C. Serum MCSF in HBV HCC patients prior to and post TACE; D. Serum MCSF in HBV HCC patients prior to and post surgery. 
Figure 3

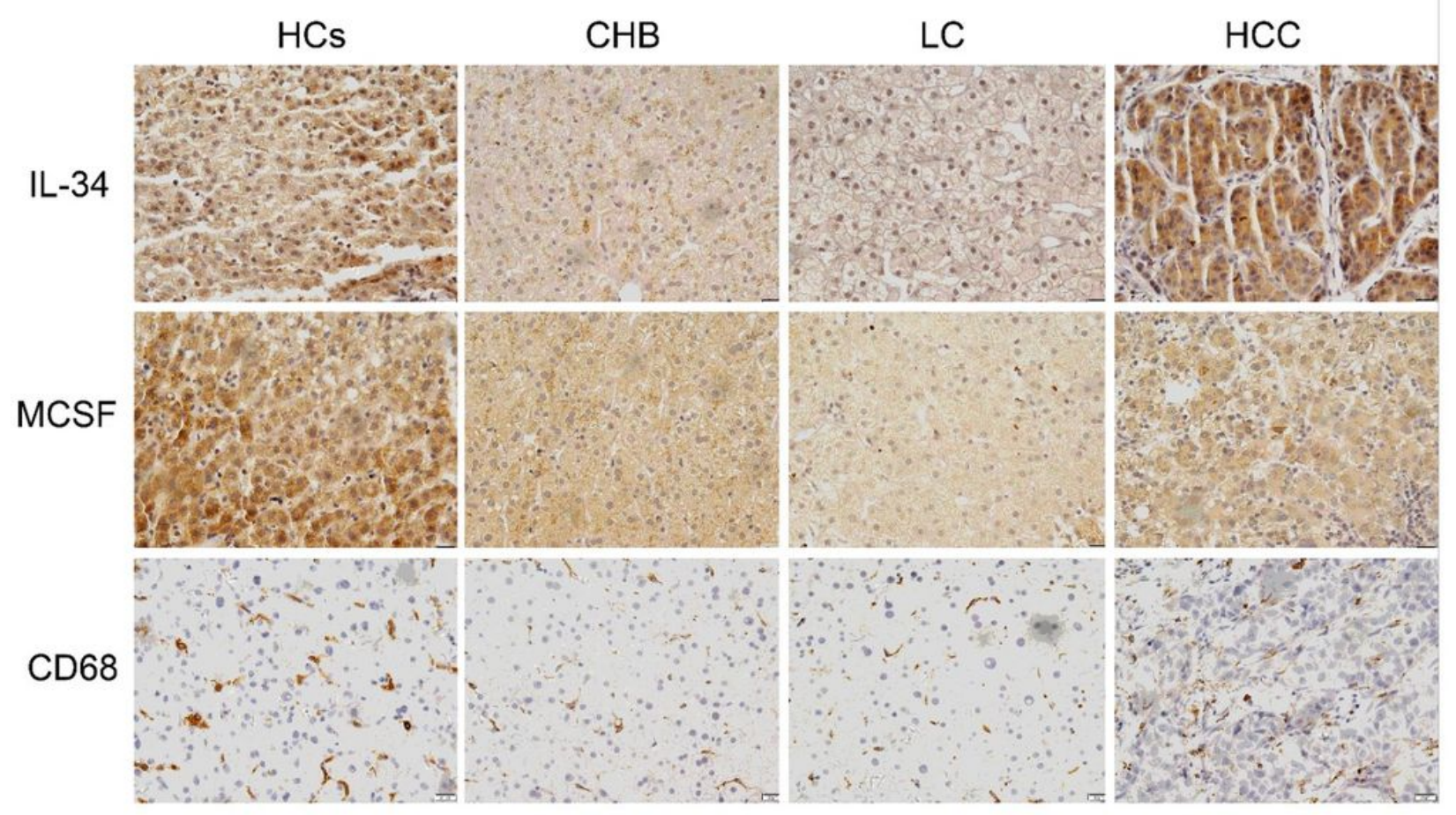

Figure 3

The immunohistochemistry of intra hepatic IL-34, MCSF, CD68+TAMs in HCs, CHB, HBV cirrhosis and HBV HCC. (LC: liver cirrhosis) 
Figure 4
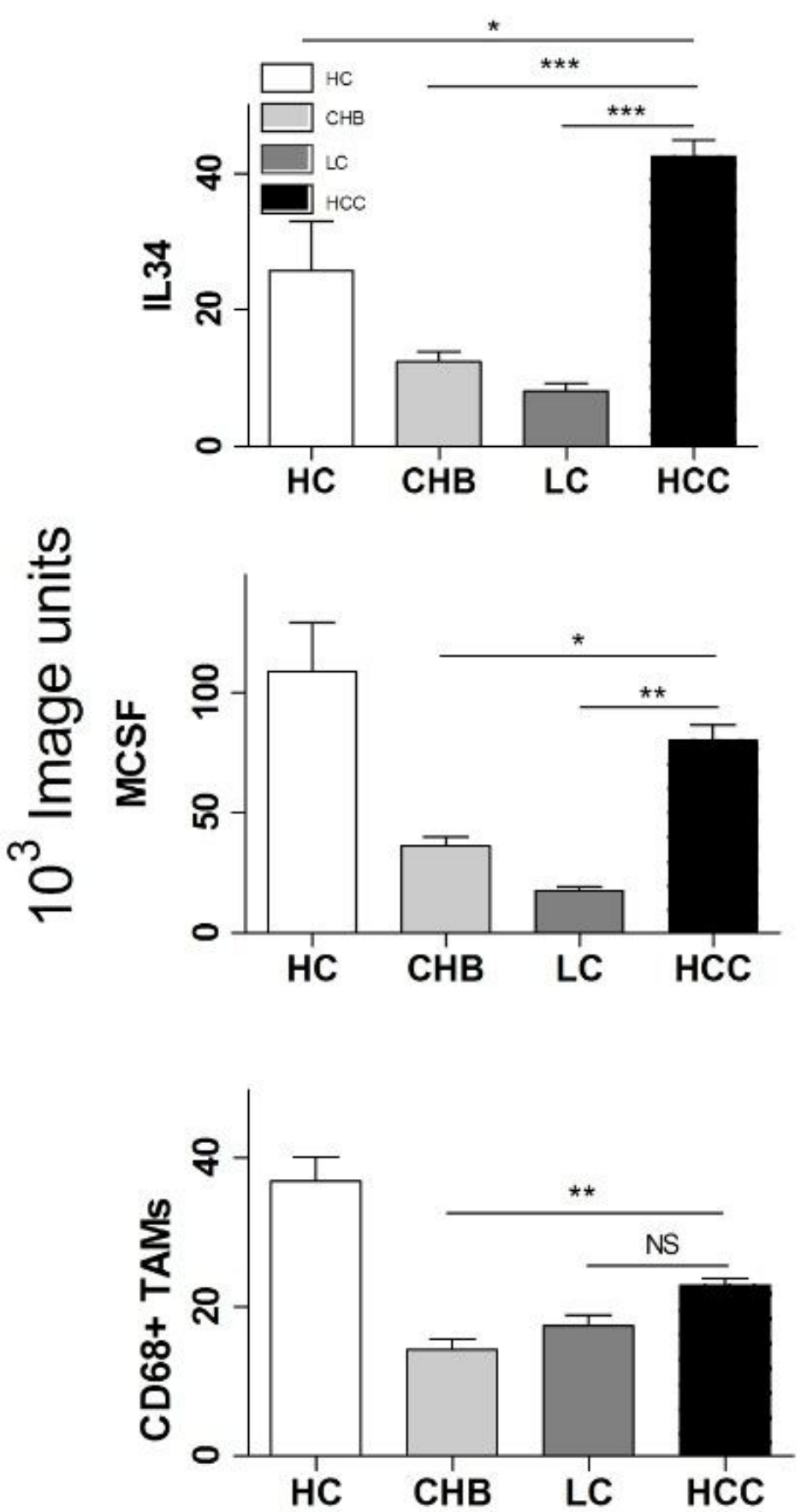

Figure 4

The corresponding quantification of immunohistochemical detection. (LC: liver cirrhosis) 
Figure 5
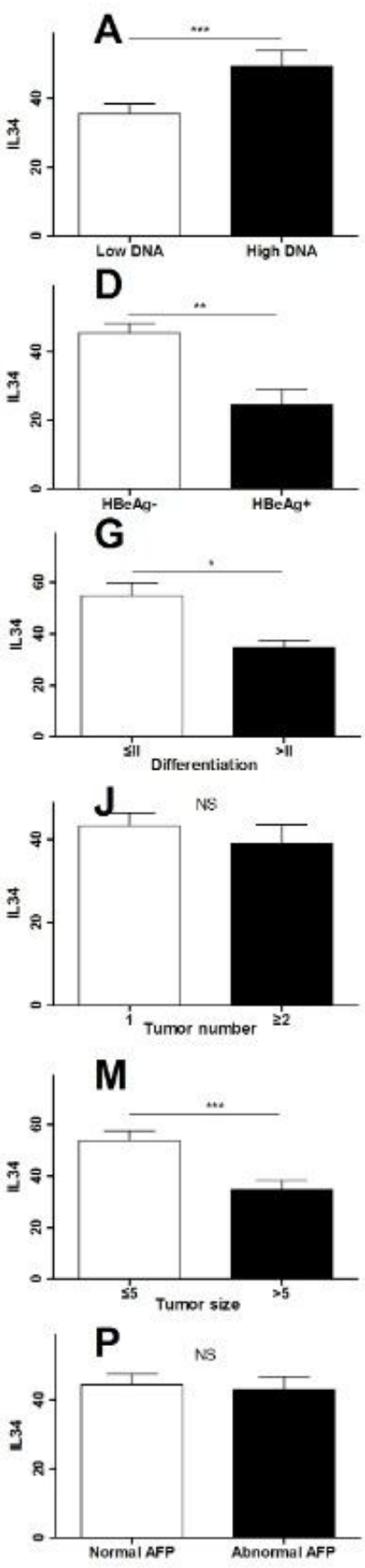

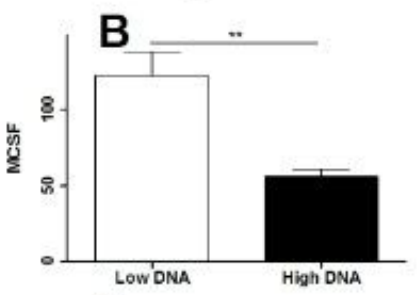

E

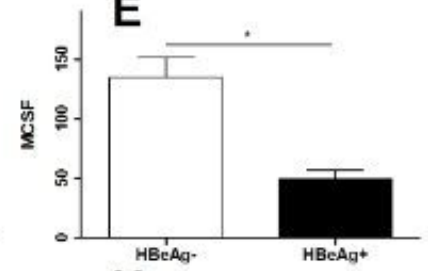

H

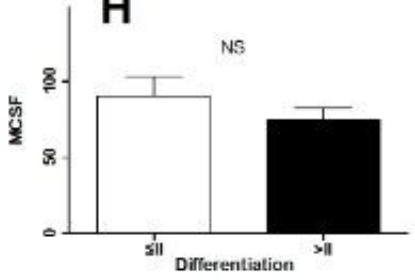

K

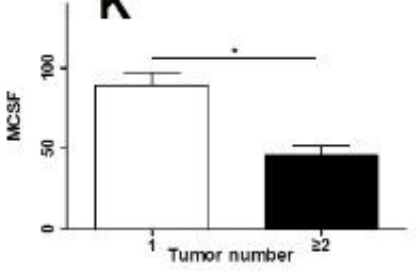

$\mathbf{N}$

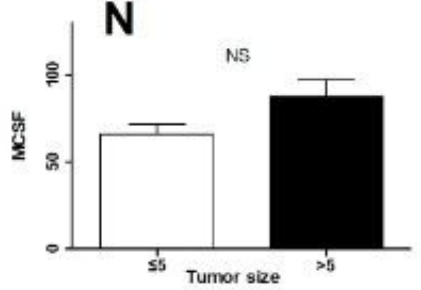

Q

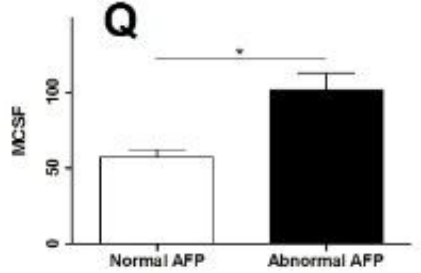

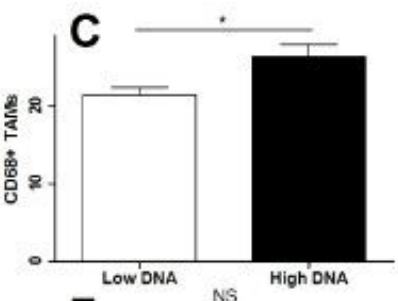
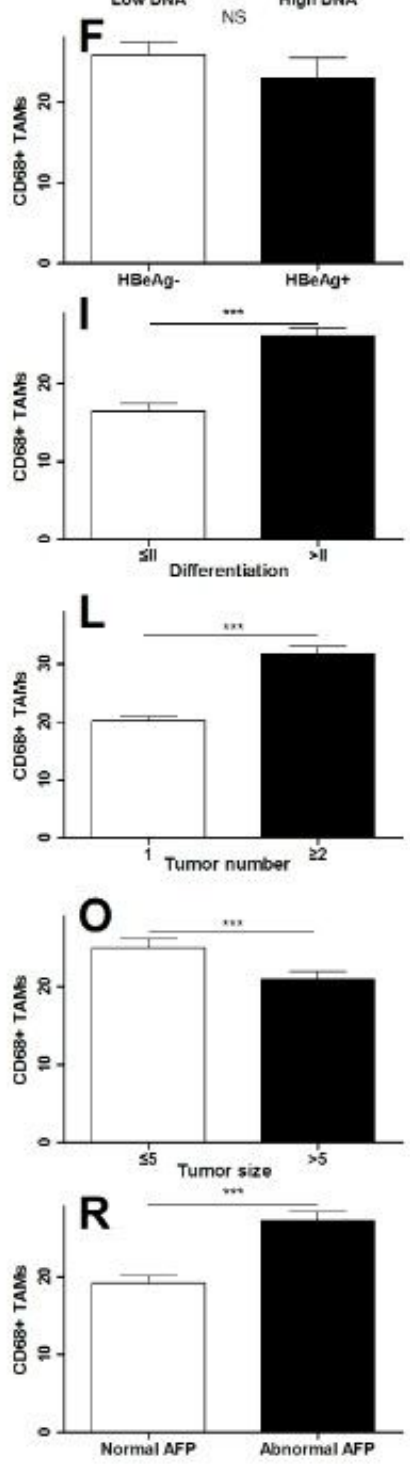

\section{Figure 5}

Correlation of intra hepatic IL 34, MCSF or CD68+ TAMs expression with HBV DNA, HBeAg and tumor differentiation subtypes. A. Correlation of intra hepatic IL 34 with HBV DNA; B. Correlation of intra hepatic MCSF with HBV DNA; C. Correlation of intra hepatic CD68+ TAMs expression with HBV DNA; D.

Correlation of intra hepatic IL 34 with HBeAg; E. Correlation of intra hepatic MCSF with HBeAg; F.

Correlation of intra hepatic CD68+ TAMs expression with HBeAg; G. Correlation of intra hepatic IL 34 with tumor differentiation; H. Correlation of intra hepatic MCSF with tumor differentiation; I. Correlation of intra 
hepatic CD68+ TAMs expression with tumor differentiation; J. Correlation of intra hepatic IL 34 with tumor number; K. Correlation of intra hepatic MCSF with tumor number; L. Correlation of intra hepatic CD68+ TAMs expression with tumor number; M. Correlation of intra hepatic IL 34 with tumor size; N. Correlation of intra hepatic MCSF with tumor size; 0 . Correlation of intra hepatic CD68+ TAMs expression with tumor size; P. Correlation of intra hepatic IL 34 with AFP; Q. Correlation of intra hepatic MCSF with AFP; R. Correlation of intra hepatic CD68+ TAMs expression with AFP. 